\title{
Long-Term Outcomes in BRAF-Mutated Melanoma Treated with Combined Targeted Therapy or Immune Checkpoint Blockade: Are We Approaching a True Cure?
}

\author{
Patrick Schummer $^{1} \cdot$ Bastian Schilling $^{1}$ (I) $\cdot$ Anja Gesierich ${ }^{1}$
}

Published online: 2 March 2020

(c) The Author(s) 2020

\begin{abstract}
Approximately $50 \%$ of all melanomas harbor an activating $B R A F$ mutation. In patients suffering from an advanced melanoma with such a somatic alteration, combined targeted therapy with a BRAF and MEK inhibitor can be applied to significantly increase the survival probability. Nevertheless, resistance mechanisms, as well as negative predictive biomarkers (elevated lactate dehydrogenase levels, high number of metastatic organ disease sites, brain metastasis), remain a major problem in treating melanoma patients. Recently, a landmark overall survival (OS) rate of $34 \%$ after 5 years of combined targeted therapy in treatment-naïve patients was reported. On the other hand, patients harboring a BRAF mutation and receiving first-line immune checkpoint blockade with ipilimumab plus nivolumab showed a 5-year OS rate of $60 \%$. As indicated by these data, long-term survival can be reached in melanoma patients but it remains unclear if this is equivalent to reaching a true cure for metastatic melanoma. In this review, we summarize the recent results for combined targeted therapy and immunotherapy in advanced melanoma harboring an activating $B R A F$ mutation and discuss the impact of baseline characteristics on longterm outcome.
\end{abstract}

\section{Key Points}

Treating BRAF V600-mutated melanomas with a BRAF and MEK inhibitor significantly increases survival outcome.

Resistance mechanisms to the BRAF and MEK inhibition still remain a major problem.

Melanoma patients harboring a $B R A F$ V600 mutation might benefit the most from a first-line immune checkpoint blockade.
Bastian Schilling

schilling_b@ukw.de

1 Department of Dermatology, University Hospital Würzburg, Josef-Schneider-Str. 2, 97080 Würzburg, Germany

\section{Introduction}

Melanoma is still one of the most severe cutaneous malignancies, with a continuously increasing incidence rate [1]. Its strong invasive capability and high level of genomic alterations account for early metastasis and deadly outcomes [2]. The identification of signaling pathways in melanoma, as well as tumor immune cell communications, have led to new therapeutic approaches in treating advanced melanoma. In particular, identification of the crucial role of the RAS-RAF-MEK-ERK (MAPK) signaling pathway can be regarded as a milestone for melanoma therapy. About half of all melanoma patients harbor an activating $B R A F$ mutation (mostly BRAF V600E), leading to increased proliferation and survival of melanoma cells [3]. Targeting this signaling pathway has led to a significant improvement in overall survival (OS) and progression-free survival (PFS), with latest results showing a landmark OS rate of $34 \%$ after 5 years of initiating such therapy in treatment-naïve patients with unresectable or metastatic melanoma [4]. Although combined targeted therapy (cTT) with a BRAF inhibitor (BRAFi) and MEK inhibitor (MEKi) is associated with a high objective response rate (ORR), most patients relapse during therapy 
due to acquired mechanisms of resistance. Several resistance mechanisms (primary and secondary) have been described $[5,6]$. Compared with BRAFi monotherapy, cTT showed fewer adverse events (AEs) and delayed occurrence of acquired resistance [7]. It is still unclear if resistance is truly acquired or represents the outgrowth of resistant clones, with the latter being more likely [8]. However, long-term PFS and OS can be observed in some patients undergoing cTT. In addition, patients harboring a $B R A F$ mutation can also receive an immune checkpoint blockade (ICB) as first-line therapy. Recent results showed a 5-year OS of $60 \%$ on ipilimumab plus nivolumab.

In this review, we summarize the latest results for patients treated with BRAF and MEK inhibitor combination therapy or ICB. In particular, we discuss baseline characteristics associated with a more favorable outcome, as well as suitable second-line therapies and strategic considerations.

\section{Progression-Free and Overall Survival of Metastatic Melanoma Patients Treated with BRAF and MEK Inhibitor Combinations}

To date, there are three US Food and Drug Administration (FDA)/European Medicines Agency (EMA)-approved $\mathrm{BRAF}$ and MEK inhibitor combination therapies for patients with advanced $B R A F$-mutated melanoma suffering from active disease: dabrafenib plus trametinib, vemurafenib plus cobimetinib and encorafenib plus binimetinib. After presenting the initial results on efficacy and safety [9-12], results from all pivotal trials were updated after additional follow-up.

\subsection{Dabrafenib Plus Trametinib (COMBI-d and $\mathrm{COMBI-v)}$}

The cTT with dabrafenib (D) and trametinib (T) has been analyzed in two prospective, randomized, controlled, phase III trials (COMBI-d, NCT01584648 [9]; and COMBI-v, NCT01597908 [10]). A 5-year pooled analysis for D+T has recently been published by Robert et al. (with a data cut-off for the COMBI-v trial of 8 October 2018, and 10 December 2018 for the COMBI-d trial) [4]. In this pooled report, 563 treatment-naïve patients with unresectable or metastatic melanoma (American Joint Committee on Cancer [AJCC] 2009 stage IIIC or IV) harboring a $B R A F \mathrm{~V} 600 \mathrm{E} / \mathrm{K}$ mutation were randomly assigned to receive either $\mathrm{D}+\mathrm{T}$ or $\mathrm{D}$ plus placebo or vemurafenib $(\mathrm{V})$. In the pooled patient cohort, the median PFS for D + T was 11.1 months (95\% confidence interval [CI] 9.5-12.8) in the intention-to-treat population. The PFS rate was $19 \%(95 \%$ CI $15-22)$ at 5 years. While patients with normal (at or below the upper limit of normal [ULN] range) lactate dehydrogenase (LDH) levels had a 5-year PFS rate of $25 \%$ (95\% CI 20-30), patients with elevated LDH levels had a 5-year PFS rate of only $8 \%$ (95\% CI 4-13). The 5-year PFS rate was higher for patients with normal LDH levels and less than three metastatic disease sites at baseline (31\%; 95\% CI 24-38). Median OS was 25.9 months (95\% CI 22.6-31.5), with a 5-year OS rate of 34\% (95\% CI 30-38). Again, significant differences in OS could be observed when baseline LDH levels and the number of metastatic organ sites were used as biomarkers. Patients with normal LDH levels showed a 5-year OS rate of $43 \%$ (95\% CI 38-49) compared with only $16 \%$ (95\% CI 11-22) for patients with elevated LDH levels at baseline. Furthermore, patients with normal LDH levels and less than three metastatic disease sites at baseline showed a 5-year OS rate of 55\% (95\% CI 48-61). Looking at the overall response rates (ORR), an objective response was documented in $68 \%$ of the pooled patients, with a complete response (CR) in 19\%. Among patients achieving a CR, the 5-year PFS rate was 49\% (95\% CI 39-58) and the OS rate was $71 \%$ (95\% CI 62-79). The median duration of CRs was 36.7 months (95\% CI 24.1-not reached), indicating that even patients with deep responses can still eventually become resistant.

\subsection{Vemurafenib Plus Cobimetinib (coBRIM)}

The coBRIM study is a prospective, randomized, doubleblind, phase III trial comparing vemurafenib (V) plus cobimetinib (C) and V plus placebo (NCT01689519 [11]). Updated efficacy results were published in 2016 by Ascierto et al. [13]. After a median follow-up of 14.2 months, the median PFS for $\mathrm{V}+\mathrm{C}$ was 12.3 months (95\% CI 9.5-13.4). A subgroup analysis for LDH levels at baseline also showed a decreased median PFS if patients had elevated LDH levels at baseline (8.2 months; 95\% CI 7.3-10.6). Patients with normal LDH levels at baseline had a higher median PFS of 13.4 months (95\% CI 12.0-16.5). Median follow-up for OS was 18.5 months, with a median OS of 22.3 months $(95 \%$ CI 20.3-not estimable). The 2-year OS rate was $48.3 \%$ (95\% CI 41.4-55.2). At data cut-off, 70\% (95\% CI 63.5-75.3) had an objective response, with $16 \%$ having a $\mathrm{CR}$. The median duration of response (DOR) in general was 13.0 months (95\% CI 11.1-16.6) and 18.1 months (95\% CI 14.8-not estimable) if the patient had a CR.

A pooled analysis of four randomized clinical trials (BRIM-2, NCT00949702; BRIM-3, NCT00949702; BRIM-7, NCT01271803; and coBRIM, NCT01689519) identified LDH levels and the sum of the longest diameters of target lesions (SLD) as significant baseline characteristics regarding survival outcome in patients receiving $\mathrm{V}+\mathrm{C}$ [14]. Patients with normal LDH levels and an SLD $\leq 45 \mathrm{~mm}$ at baseline had a median OS of 27.2 months $(95 \% \mathrm{CI}$ 22.1-32.1) with $\mathrm{V}+\mathrm{C}$. In contrast, if patients had elevated 
LDH levels ( $\geq 2 \times \mathrm{ULN})$, the median OS was 6.0 months (95\% CI 5.3-6.8). The 3-year landmark OS was 53.3\% (95\% CI 39.5-67.1) and 8.8\% (95\% CI 0.0-18.4), respectively.

\subsection{Encorafenib Plus Binimetinib (COLUMBUS)}

Another cTT consisting of the BRAFi encorafenib (E) and the MEKi binimetinib (B) has been analyzed in the COLUMBUS trial, a two-part, randomized, open-label, phase III study (NCT01909453 [12, 15]). In part 1, the combination of $\mathrm{E}+\mathrm{B}$ was compared with $\mathrm{V}$ monotherapy. The median follow-up for OS was 36.8 months, with a median OS for $\mathrm{E}+\mathrm{B}$ of 33.6 months, compared with 16.9 months for $\mathrm{V}$ (hazard ratio [HR] $0.61,95 \% \mathrm{CI} 0.47-0.79 ; p<0.0001$ ). The 2-year OS rate for $\mathrm{E}+\mathrm{B}$ was $57.6 \%$. Median follow-up for PFS was 32.1 months, with a median PFS of 14.9 months for $\mathrm{E}+\mathrm{B}$ versus 7.3 months for $\mathrm{V}$ (HR $0.51,95 \%$ CI 0.39-0.67; $p<0.0001)$. Confirmed ORR was observed in $64 \%$ of $\mathrm{E}+\mathrm{B}$ patients. At data cut-off, CR occurred in $11 \%$ of patients receiving a cTT with $\mathrm{E}+\mathrm{B}$. Regarding OS subgroup analyses, the cTT with E+B was favored in most but not all subgroups (e.g. not in patients with elevated LDH levels at baseline). Recently, Liszkay et al. presented updated efficacy results for E + B at the annual 2019 American Society of Clinical Oncology (ASCO) meeting, however these have not yet been published. In that abstract, the median OS for $\mathrm{E}+\mathrm{B}$ was 33.6 months (95\% CI 24.4-39.2) after a median followup of 48.6 months. The median PFS was 14.9 months (95\% CI 11.0-20.2), compared with 7.3 months (95\% CI 5.6-8.2) for V monotherapy. The 4-year landmark PFS and OS for $\mathrm{E}+\mathrm{B}$ was $25 \%$ and $39 \%$, respectively.

\section{Efficacy of Immune Checkpoint Blockade (ICB) in Patients with Advanced Melanoma Harboring a BRAF V600 Mutation}

Apart from targeted therapies (TTs), patients with a $B R A F$ mutation can undergo ICB. The following section summarizes the latest results for the different therapeutic options.

\subsection{Anti-PD-1 Monotherapy (Pembrolizumab, Nivolumab)}

Anti-programmed cell death protein 1 (PD-1) monotherapies show significant activity in metastatic melanoma, regardless of $B R A F$ status. A recently published 5-year survival analysis of the Keynote- 001 trial, a phase $1 \mathrm{~b}$ study (NCT01295827), showed a 5-year OS rate of 34\% in all patients treated with pembrolizumab after a median followup of 55 months [16]. Treatment-naïve patients showed a notably higher 5 -year OS rate of $41 \%$. The median OS for each group was 23.8 months (95\% CI 20.2-30.2) and
38.6 months (95\% CI 27.2-not reached), respectively. In this cohort, only $B R A F$ wild-type patients had been treatmentnaïve, since patients with a $B R A F$ mutation had to have had previous TT. Another study evaluating the efficacy of pembrolizumab in BRAF V600-mutated melanoma patients (Keynote-006, NCT01866319) also showed a promising 5-year median OS of 32.7 months (95\% CI 24.5-41.6) for pembrolizumab, with a median follow-up of 57.7 months, but $B R A F$ status-stratified OS was not reported [17].

Based on these data, BRAF status-stratified median OS in patients receiving pembrolizumab cannot be reported. For nivolumab, survival analyses of two phase III trials have been published. First, the CheckMate 067 trial (NCT01844505) showed a 5-year landmark OS rate of 44\%, with a median OS of 36.9 months (95\% CI 28.2-58.7) for nivolumab in the entire study population [18], while the 5 -year landmark PFS rate was $29 \%$, with a median PFS of 6.9 months (95\% CI 5.1-10.2). In the BRAF-mutated subgroup, a 5-year landmark OS and PFS rate of $46 \%$ and $22 \%$, respectively, was observed. The median OS was 45.5 months (95\% CI 26.4-not reached) and the median PFS was 5.6 months (95\% CI 2.8-9.5). A possible explanation for the better median OS could be due to subsequent therapies, e.g. cTT. Second, the CheckMate 037 trial (NCT01721746) evaluated nivolumab in advanced melanoma patients who progressed under ipilimumab therapy and, if $B R A F$-mutated, BRAFi therapy also [19]. The median OS was 15.7 months (95\% CI 12.9-19.9), with a 2-year OS rate of $38.7 \%$ (95\% CI 32.8-44.5) in the entire trial population. In this trial, $20 \%$ of the nivolumab patients had brain metastases and 52\% had elevated LDH levels at baseline, which still shows a promising survival outcome due to worse baseline characteristics. Prespecified subgroup analyses showed no notable differences in OS. No specific OS was reported for $B R A F$-mutated patients in this trial.

\subsection{Combined ICB with Ipilimumab Plus Nivolumab}

After a minimal follow-up of 5 years, the combination ICB of ipilimumab plus nivolumab demonstrated a 5-year OS rate of $52 \%$ in all treatment-naïve patients with advanced melanoma enrolled in CheckMate 067 (NCT01844505) [18]. Subgroup analyses showed a slightly better outcome for patients harboring a $B R A F$ mutation compared with those without, with a 5-year OS rate of $60 \%$ and $46 \%$, respectively. The median PFS was 11.5 months (95\% CI 8.7-19.3), with a 5-year PFS rate of $36 \%$ in the entire study population. For $B R A F$-mutated patients, the median PFS was 16.8 months (95\% CI 8.3-32.0), with a 5-year PFS rate of 38\%. In contrast, $B R A F$ wild-type patients showed a median PFS of 11.2 months (95\% CI 7.0-18.1) and a 5-year PFS rate of $35 \%$. The ORR for ipilimumab plus nivolumab was $58 \%$, with CR rates of $22 \%$ in all patients. The $14 \%$ difference in 
the 5-year OS rate defined by $B R A F$ status is accompanied by only a small difference in the 5-year PFS rate, indicating an impact of second-line therapy. The indirect comparison indicates that ipilimumab plus nivolumab first-line followed by cTT (if necessary) is the more promising strategy in advanced BRAFV600-mutated melanoma.

Table 1 summarizes the baseline characteristics, median PFS and OS, and the highest and lowest 3-year PFS/OS data for each pivotal cTT trial and for the combined ICB trial (CheckMate 067).

\section{Which Melanoma Patients Show a More Favorable Treatment Outcome?}

There are some baseline characteristics associated with a more favorable or inferior outcome for melanoma patients treated with cTTs or ICB first-line. In particular, elevated LDH levels at baseline, an Eastern Cooperative Oncology Group performance status (ECOG PS) $>0$, and a higher number of metastatic organ sites correlate with shorter PFS and OS [4, 18, 20, 21]. However, an indirect comparison of the cTT and ICB regimens is very difficult due to differences in the patient populations and the biomarkers reported.

\subsection{Serum Lactate Dehydrogenase}

Hauschild et al. performed a pooled analysis of four randomized clinical trials (BRIM-2, NCT00949702; BRIM-3, NCT00949702; BRIM-7, NCT01271803; and coBRIM, NCT01689519) and, by using a recursive partitioning decision tree, identified baseline LDH levels, ECOG PS, and disease burden as crucial determinants of survival outcomes for patients treated with cTT [14]. Patients with normal LDH levels at baseline and without liver metastases showed a median 3-year PFS of 26.9 months (95\% CI 16.1-37.8) on $\mathrm{V}+\mathrm{C}$. In comparison, patients with elevated LDH levels at baseline ( $\geq 2 \times \mathrm{ULN})$ had a median PFS of only 2.9 months (95\% CI 0.0-8.4). The ORR ranged from $54.3 \%$ in patients with the poorest prognostic characteristic ( $\mathrm{LDH}$ levels $\geq 2 \times \mathrm{ULN}$ ) to $77.1 \%$ for patients with the most favorable characteristics (normal LDH levels, absence of liver metastases). A CR occurred in $30.5 \%$ of patients with normal LDH levels and absence of liver metastases at baseline, compared with $2.9 \%$ of patients with elevated LDH levels $(\geq 2 \times U L N)$. For $D+T$, the pooled analysis by Robert et al. of the COMBI-d and COMBI-v trials showed a similar survival outcome regarding baseline LDH levels [4]. At 5 years, patients with normal LDH levels at baseline showed a PFS rate of $25 \%$ (95\% CI 20-30), compared with $8 \%$ (95\% CI 4-13) for patients with elevated LDH levels at baseline. In comparison to the pooled analysis by Hauschild et al., which detected the presence/absence of liver metastasis as a prognostic factor, Robert et al. presented higher PFS rates for patients with less than three metastatic disease sites in addition to normal LDH levels at baseline (5-year PFS rate 31\%; 95\% CI 24-38). For E+B, no respective data for patients with elevated LDH levels at baseline are available yet.

Baseline LDH levels are also of great importance regarding the survival outcome of patients undergoing ICBs. Recently updated 5-year results of the CheckMate 067 trial (NCT01844505) showed a median OS of > 60.0 months (95\% CI not reached-not reached) for patients with normal baseline LDH levels treated with ipilimumab plus nivolumab [18]. In comparison, patients with elevated baseline LDH levels (LDH levels $>$ ULN) presented a median OS of only 17.4 months (95\% CI 10.7-42.6). The median PFS was 21.2 months (95\% CI 11.5-47.1) and 4.2 months (95\% CI 2.8-9.3), respectively. In the nivolumab monotherapy cohort, the median OS was > 60.0 months (95\% CI 40.2-not reached) with normal LDH levels at baseline, compared with 16.0 months (95\% CI 11.7-21.6) with elevated (> ULN) LDH levels at baseline. The respective median PFS rates were 12.4 months (95\% CI 6.9-25.6) and 2.8 months $(95 \%$ CI 2.6-4.0). Of note, these survival outcomes stratified by LDH level included all patients irrespective of $B R A F$ status. At the time of data collection, no respective subgroup analysis for BRAF V600-mutated patients only had been performed.

\subsection{Melanoma Brain Metastases}

Another crucial characteristic regarding the survival outcome of melanoma patients is the presence of melanoma brain metastases (MBMs) [22]. All pivotal cTT trials (COMBI-d/v, coBRIM, COLUMBUS) either excluded patients with a known history of MBMs or only included them if the MBMs were asymptomatic and had been previously treated. Therefore, prospective data on the efficacy of cTTs in melanoma patients with active MBMs is limited but available. One trial evaluating $\mathrm{D}+\mathrm{T}$ in melanoma patients with known MBMs is the COMBI-MB study (NCT02039947), a phase II, open-label trial [23]. Here, a total of 125 patients have been assigned into four cohorts: (A) BRAF V600E, asymptomatic MBMs, no prior local brain therapy $(n=76)$; (B) $B R A F \mathrm{~V} 600 \mathrm{E}$, asymptomatic MBMs, prior local brain therapy $(n=16)$; (C) BRAF V600D/ $\mathrm{K} / \mathrm{R}$, asymptomatic MBMs, with or without prior local brain therapy ( $n=16$ ); and (D) $B R A F \mathrm{~V} 600 \mathrm{D} / \mathrm{E} / \mathrm{K} / \mathrm{R}$, symptomatic MBMs, with or without prior local brain therapy $(n=17)$. At data cut-off (28 November 2016) with a median follow-up of 8.5 months, the intracranial response rates (IRR) were $58 \%$ for cohort A, $56 \%$ for cohort B, $44 \%$ for cohort C and $59 \%$ for cohort D. The preliminary median OS for cohorts A-D was 10.8 months (95\% CI 8.7-19.6), 24.3 months (95\% 
Table 1 Patient characteristics in the cTT arms of pivotal trials and the ipilimumab plus nivolumab group of the CheckMate 067 trial

\begin{tabular}{|c|c|c|c|c|c|}
\hline & COMBI-d (D+T) [56] & COMBI-v $(\mathrm{D}+\mathrm{T})[10]$ & coBRIM $(\mathrm{V}+\mathrm{C})[13]$ & $\begin{array}{l}\text { COLUMBUS } \\
\text { Part } 1(E+B) \\
{[15]}\end{array}$ & $\begin{array}{l}\text { CheckMate } 067(\mathrm{I}+\mathrm{N}) \\
{[58]}\end{array}$ \\
\hline $\begin{array}{l}\text { Median age, years } \\
\text { (range) }\end{array}$ & $55(22-89)$ & $55(18-91)$ & $56(23-88)$ & $58(20-89)$ & $61(18-88)$ \\
\hline \multicolumn{6}{|l|}{ Sex } \\
\hline Men & 53 & 59 & 59 & 60 & 66 \\
\hline Women & 47 & 41 & 41 & 40 & 34 \\
\hline \multicolumn{6}{|l|}{ ECOG PS } \\
\hline 0 & 74 & 71 & 76 & 71 & 73 \\
\hline 1 & 26 & 29 & 24 & 29 & 26 \\
\hline \multicolumn{6}{|l|}{ M stage } \\
\hline IIIC & 3 & 4 & 9 & 5 & $42^{\mathrm{a}}$ \\
\hline M1a & 9 & 16 & 16 & 14 & \\
\hline M1b & 21 & 17 & 16 & 18 & \\
\hline M1c & 67 & 63 & 59 & 64 & 58 \\
\hline $\mathrm{LDH}>\mathrm{ULN}$ & 37 & 34 & 46 & 29 & 36 \\
\hline $\begin{array}{l}\text { History of brain metas- } \\
\text { tases }\end{array}$ & NA & NA & $<1$ & NA & 4 \\
\hline \multicolumn{6}{|l|}{$\begin{array}{l}\text { BRAF mutation } \\
\text { subtype }\end{array}$} \\
\hline V600E & 85 & 90 & $69^{c}$ & 89 & $32^{\mathrm{b}}$ \\
\hline V600K & 15 & 10 & 10 & 11 & \\
\hline mPFS, months & 11.0 & 11.4 & 12.3 & 14.9 & 11.5 \\
\hline mOS, months & 25.1 & NR & 22.3 & 33.6 & NR \\
\hline ORR & 69 & 64 & 70 & 63 & 57.6 \\
\hline $\mathrm{CR}$ & 16 & 13 & 10 & 11 & 11.5 \\
\hline $\begin{array}{l}\text { Highest 3-year PFS rate } \\
\text { (associated baseline } \\
\text { biomarkers) }\end{array}$ & \multicolumn{2}{|c|}{39 (normal LDH, $<3$ disease sites) $^{\mathrm{d}}$} & $\begin{array}{l}51.8 \text { (normal LDH, } \\
\text { SLD } \leq 44 \mathrm{~mm}, \text { ECOG } \\
\text { PS 0) }\end{array}$ & NA & $45(\mathrm{LDH} \leq \mathrm{ULN})^{\mathrm{f}}$ \\
\hline $\begin{array}{l}\text { Highest } 3 \text {-year OS rate } \\
\text { (associated baseline } \\
\text { biomarkers) }\end{array}$ & \multicolumn{2}{|c|}{ 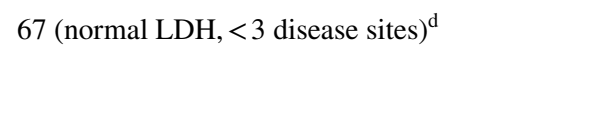 } & $\begin{array}{l}53.3(\text { normal LDH, } \\
\mathrm{SLD} \leq 44 \mathrm{~mm})^{\mathrm{e}}\end{array}$ & NA & 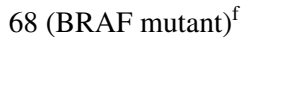 \\
\hline $\begin{array}{l}\text { Lowest 3-year PFS rate } \\
\text { (associated baseline } \\
\text { biomarkers) }\end{array}$ & \multicolumn{2}{|l|}{$9(\mathrm{LDH}>\mathrm{ULN})^{\mathrm{d}}$} & NA & NA & $17(\mathrm{LDH}>2 \times \mathrm{ULN})^{\mathrm{f}}$ \\
\hline $\begin{array}{l}\text { Lowest 3-year OS rate } \\
\text { (associated baseline } \\
\text { biomarkers) }\end{array}$ & \multicolumn{2}{|l|}{$22(\mathrm{LDH}>\mathrm{ULN})^{\mathrm{d}}$} & $8.8(\mathrm{LDH} \geq 2 \mathrm{ULN})^{\mathrm{e}}$ & NA & $31(\mathrm{LDH}>2 \times \mathrm{ULN})^{\mathrm{f}}$ \\
\hline
\end{tabular}

Data are expressed as percentages unless otherwise specified

$B$ binimetinib, $C$ cobimetinib, $C R$ complete response, $c T T$ combined targeted therapy, $D$ dabrafenib, $E$ encorafenib, ECOG PS Eastern Cooperative Oncology Group performance status, $I$ ipilimumab, $L D H$ lactate dehydrogenase, $m O S$ median overall survival, $m P F S$ median progressionfree survival, $N$ nivolumab, $N A$ not available, $N R$ not reached, $O R R$ objective response rate, $P F S$ progression-free survival, $S L D$ sum of the longest diameters of target lesions, $T$ trametinib, $U L N$ upper limit of normal, $V$ vemurafenib

${ }^{\mathrm{a}}$ This is the percentage for M stage IIIc, M1a and M1b combined

${ }^{\mathrm{b}} \mathrm{This}$ is the percantage for $\mathrm{V} 600 \mathrm{E}$ and $\mathrm{V} 600 \mathrm{~K}$ combined

${ }^{\mathrm{c}}$ In $22 \%$ of patients, no tissue was tested by the sponsor, but locally

${ }^{\mathrm{d}}$ Three-year landmark data acquired from the pooled analysis of D+T by Robert et al. [4]

${ }^{\mathrm{e}}$ Three-year landmark data acquired from the pooled analysis of $\mathrm{V}+\mathrm{C}$ by Hauschild et al. [14]

${ }^{\mathrm{f}}$ Three-year landmark data acquired from the updated efficacy analysis by Wolchok et al. [55] 
CI 7.9-not estimated), 10.1 months (95\% CI 4.6-17.6), and 11.5 months (95\% CI 6.8-22.4), respectively. Besides the promising IRR, the DOR still remains lower compared with extracranial disease sites. In this trial, cohort A showed a median intracranial DOR of 6.5 months (95\% CI 4.9-10.3), compared with a median extracranial DOR of 10.2 months (95\% CI 5.8-not estimable). The 3-year landmark analysis of the COMBI-d trial showed a similar median extracranial DOR of 12.0 months (95\% CI 9.3-17.1), underlining a crucial impact of MBMs on survival outcome [23]. At the time of data collection, only case reports and/or non-randomized retrospective data have been available regarding the survival outcome of melanoma patients with MBMs treated with the cTTs $\mathrm{V}+\mathrm{C}$ or $\mathrm{E}+\mathrm{B}$.

Furthermore, treating MBMs with ICB also shows encouraging results. While MBMs were an exclusion criteria in CheckMate 067, two multicenter, phase II trials have analyzed the combination ICB (ipilimumab plus nivolumab) in melanoma patients with asymptomatic MBMs. In the ABC study (NCT02374242), 35 patients received a combination ICB [24]. No prior local brain therapy was allowed, whereas patients with intracranial disease progression on BRAFi therapy could have been included. In this trial, $23 \%$ $(n=8 / 35)$ of patients had received a previous cTT. After a median follow-up of 17 months, 46\% (95\% CI 29-63) of all patients showed an intracranial response, with $17 \%$ receiving an intracranial CR. In this trial, drug-naïve patients $(n=27 / 35)$ showed a higher treatment response, with an overall IRR of 56\% (95\% CI 35-75) and 19\% received an intracranial CR. Due to the small patient population, these data should be considered cautiously. In addition, the CheckMate 204 trial (NCT02320058) detected an IRR of 57\% (95\% CI 47-68), with an intracranial CR of $26 \%$ after a median follow-up of 14.0 months [25]. In this trial, $57.4 \%$ of patients harbored a $B R A F$ mutation and $41.5 \%$ had elevated ( $>\mathrm{ULN}$ ) LDH levels at baseline. Prior targeted therapy has been allowed for $B R A F$-mutated patients. The objective IRR was in favor of an existing $B R A F$ mutation, i.e. $57.4 \%(95 \%$ CI 43.2-70.8) for patients with a BRAF mutation, compared with $40.0 \%$ (95\% CI 21.2-61.3) for patients without a BRAF mutation.

\subsection{Depth of Response}

Best overall response (BOR) to cTT is also relevant for the long-term outcome. As Robert et al. demonstrated in the 5 -year pooled analysis for $\mathrm{D}+\mathrm{T}$, patients with a $\mathrm{CR}$ showed 5 -year OS rates of $71 \%$ (95\% CI 62-79) [4]. In contrast, patients with stable disease (SD) as the BOR only had a 5 -year OS rate of $16 \%$ (95\% CI 10-24). Of the 161 patients who remained on study and were alive after 5 years, $45 \%$ had a CR, compared with $9 \%$ with SD and $<1 \%$ with progressive disease (PD) as the BOR. For $\mathrm{V}+\mathrm{C}$, achieving a
$\mathrm{CR}$ is associated with a high median DOR (mDOR). At the data cut-off date for the coBRIM trial (16 January 2015), $16 \%$ of patients had a CR, $18 \%$ had SD, and $8 \%$ had PD as the BOR [13]. The mDOR was 13.0 months $(95 \% \mathrm{CI}$ 11.1-16.6) in all patients and 18.1 months (95\% CI 14.8-not estimable) for patients with a CR as the BOR. At the time of data collection, no such subgroup analysis for $\mathrm{E}+\mathrm{B}$ has been available. In addition, another pooled analysis of four randomized clinical trials (BRIM-2, BRIM-3, BRIM-7, and coBRIM) demonstrated a better outcome for patients with a deep response to TT [26]. Patients had been categorized into quartiles (Q1-Q4) depending on the maximal tumor reduction (Max\%SLD), described as the greatest reduction or minimum increase in the SLD from baseline to the first progression of disease (PD) or date of last follow-up. At data cut-off for PFS and OS analysis (10 July 2017 for BRIM-7 and 13 October 2017 for coBRIM), median PFS and OS data had not been reached for Q1 $(n=73 / 310$; -100.0 to $-91.7 \%$ tumor reduction). Patients with $\mathrm{V}$ monotherapy showed a median OS of 32.1 months $(95 \%$ CI 25.9-42.5) if having a deep response-quartile Q1 $(n=171 / 717 ;-100.0$ to $-71.1 \%$ tumor reduction). In contrast, the median OS was 9.4 months (95\% CI 7.9-11.3) for a poor response or tumor progression to $\mathrm{V}$ monotherapy-quartile Q4 ( $n=170 / 717 ;-28.1$ to $+177.8 \%$ tumor reduction).

\subsection{BRAF Mutations Other than or in Addition to V600}

Regarding $B R A F$ mutational status, all four of the described cTT trials (COMBI-d, COMBI-v, coBRIM, and COLUMBUS) only included patients harboring a $B R A F \mathrm{~V} 600 \mathrm{E} / \mathrm{K}$ mutation, yet less frequent $B R A F$ mutations have been described. Recently, Menzer et al. analyzed the clinical outcomes of 103 patients harboring rare BRAF mutations or translocations [27]. Of these patients, seven had been excluded from statistical analysis due to a co-existing $B R A F \mathrm{~V} 600 \mathrm{E} / \mathrm{K}$ mutation. Of the remaining 96 patients, $58 \%$ received BRAFi/MEKi combination therapy; $76 \%$ of the patients were treatment-naïve, whereas the remainder had prior treatment that mostly consisted of immunotherapy and chemotherapy. Elevated LDH levels at baseline were found in $42 \%$ of patients. The assessed 96 patients were split into two groups, depending on a $B R A F$ V60 mutation (other than $\mathrm{V} 600 \mathrm{E} / \mathrm{K}, n=58$ ) and $B R A F$ mutations affecting other codons (e.g. K601E and L597P/Q/R/S, $n=38$ ) or $B R A F$ chromosome translocations resulting in a mutational activation of BRAF. OS for patients harboring a V600 mutation was 17.3 months (95\% CI 12.3-not available [NA]), compared with 11.3 months (95\% CI 3.8-NA) for non-V600 mutations if treated with cTT. The median PFS was 8.0 months (95\% CI 5.1-15.0) for V600 mutations and 3.3 months (95\% CI 2.2-NA) for non-V600 mutations. 
The ORR was $56 \%$ and $28 \%$, respectively. Although only a small number of patients were included, activating mutations in $B R A F$ outside of codon 600 seem to be associated with decreased efficacy of cTT.

Table 2 summarizes the biomarkers, which seem to have an impact on PFS, according to the respective cTT trials previously mentioned and prospective ICB clinical trials. Due to the lack of published data, E+B was excluded from this summary.

\section{Resistance Mechanisms to Combined BRAF and MEK Inhibition and ICB Remain a Major Problem in Melanoma Therapy}

Looking at the long-term data of each cTT study (COMBId, COMBI-V, coBRIM, and COLUMBUS), most patients progress after approximately 12 months of therapy due to acquired resistance. Primary resistance to TT, i.e. direct lack of therapeutic response, occurs less frequently. There are many possible ways described in the literature regarding resistance mechanisms, one of which is alternative activation of the MAPK signaling pathway due to different receptor tyrosine kinases, e.g. EGFR, PDGFRB, and FGFR1 [28-31]. Additionally, secondary mutations in the $B R A F$ gene, as well as alternative splicing, have also been identified [32, 33]. Furthermore, patients harboring an activating NRAS mutation also showed higher rates of BRAFi/MEKi resistance, mostly due to activation of the PI3K/AKT signaling pathway $[30,34]$. Other mutations of the PI3K/AKT signaling pathway have also been associated with a resistance to BRAFi/
MEKi, e.g. PTEN loss-of-function mutations [35]. Although of diverse nature, all mechanisms share one particular feature-they are unbreakable at this point in time. Unfortunately, cross-resistance to immune checkpoint inhibitions seems to exist on the molecular level [36].

Hugo et al. identified a transcriptomic signature with an upregulation of genes involved in cell adhesion, mesenchymal transition, angiogenesis, and extracellular matrix remodeling (e.g. AXL, VEGFA, WNT5A, IL10, CCL7) in patients not responding to first-line anti-PD-1 therapy [37]. These transcriptomic signatures are associated with an innate antiPD-1 resistance (IPRES). Similar gene upregulation could also be found after MAPK inhibition, suggesting a possible cross-resistance to a subsequent anti-PD-1 therapy.

Kreft et al. demonstrated that patients who have progressed on cTT show a less favorable outcome after second-line ICB [38]. Here, an objective response (partial or complete response) was achieved in $18.0 \%(n=7 / 39)$ of patients receiving second-line anti-PD-1 monotherapy (either nivolumab or pembrolizumab) and $15.0 \%(n=9 / 60)$ of patients receiving a combined ICB (ipilimumab plus nivolumab). The ORR for patients without MBMs at baseline was $26.4 \%(n=5 / 19)$ and $22.2 \%(n=6 / 27)$, respectively. For patients with MBMs prior to second-line ICB, the respective ORRs were $10.0 \%(n=2 / 20)$ and $9.1 \%$ $(n=3 / 33)$. Additionally, Mason et al. also showed a less favorable survival outcome for patients receiving a combination ICB (ipilimumab plus nivolumab) after not responding to first-line TT compared with first-line combination ICB, with a median PFS of 2.0 months (95\% CI 1.4-4.6) and 11.0 months (95\% CI 6.0-not reached), respectively, as well

Table 2 Baseline characteristics (biomarkers) affecting the PFS of cTT or ICB in pivotal trials

\begin{tabular}{|c|c|c|c|}
\hline & Favorable PFS if & Unfavorable PFS if & Prognostic biomarkers \\
\hline $\mathrm{D}+\mathrm{T}[4]$ & $\begin{array}{l}\mathrm{LDH} \leq \mathrm{ULN} \\
\text { BRAF V600E } \\
\text { Older age } \\
\text { Female sex } \\
\text { ECOG PS }=0 \\
\leq 3 \text { disease sites }\end{array}$ & $\begin{array}{l}\text { LDH }>\text { ULN } \\
\text { BRAF V600K } \\
\text { Younger age } \\
\text { Male sex } \\
\text { ECOG PS >0 } \\
>3 \text { disease sites }\end{array}$ & MBMs, LDH, ECOG \\
\hline $\mathrm{V}+\mathrm{C}[14]$ & $\begin{array}{l}\mathrm{LDH} \leq \mathrm{ULN} \\
\mathrm{ECOG} \mathrm{PS}=0 \\
\mathrm{SLD} \leq 44 \mathrm{~mm} \\
\text { Absence of liver metastases }\end{array}$ & $\begin{array}{l}\mathrm{LDH} \geq 2 \times \mathrm{ULN} \\
\mathrm{ECOG} \geq 1 \\
\text { SLD }>44 \mathrm{~mm} \\
\text { Presence of liver metastases }\end{array}$ & \\
\hline $\begin{array}{l}\text { PD-1 blockade (nivolumab, pembroli- } \\
\text { zumab) }[18,19,59,60]\end{array}$ & $\begin{array}{l}\text { PD-L } 1 \geq 5 \% \\
\mathrm{LDH} \leq \mathrm{ULN} \\
\mathrm{ECOG} \mathrm{PS}=0\end{array}$ & $\begin{array}{l}\text { PD-L } 1<5 \% \\
\text { LDH }>2 \times \text { ULN } \\
\text { ECOG PS }>0\end{array}$ & \\
\hline Ipilimumab plus nivolumab [18] & $\begin{array}{l}\mathrm{LDH} \leq \mathrm{ULN} \\
\leq 3 \text { disease sites } \\
\mathrm{PD}-\mathrm{L} 1 \geq 1 \%\end{array}$ & $\begin{array}{l}\mathrm{LDH}>2 \times \mathrm{ULN} \\
>3 \text { disease sites } \\
\mathrm{PD}-\mathrm{L} 1<1 \%\end{array}$ & \\
\hline
\end{tabular}

$C$ cobimetinib, $c T T$ combined targeted therapy, $D$ dabrafenib, ECOG PS Eastern Cooperative Oncology Group performance status, $I C B$ immune checkpoint blockade, $L D H$ lactate dehydrogenase, $M B M s$ melanoma brain metastases, $P D-1$ programmed cell death protein $1, P D-L 1$ programmed cell death ligand 1, PFS progression-free survival, $S L D$ sum of the longest diameters of target lesions, $T$ trametinib, $U L N$ upper limit of normal, $V$ vemurafenib 
as an ORR of $21 \%$ in BRAFi/MEKi failure patients [39]. In agreement, another retrospective study also identified lower response rates for patients treated with second-line ICB (exclusively ipilimumab) after progression on TT compared with patients with second-line TT after progression on ICB [40]. The median PFS and OS for patients receiving ipilimumab after tumor progression to TT was 2.7 months (95\% CI 1.8-3.1) and 5.0 months (95\% CI 3.0-8.8), respectively. Another study also demonstrated a reduced response rate to ICB after progression with cTT [41]. Here, 52/100 patients treated with first-line cTT received a second-line ICB monotherapy (nivolumab or pembrolizumab) after tumor progression, of whom only 4/52 showed an objective response, whereas 36/52 had PD as their BOR.

These data indicate activity of anti-PD-1-based ICB in patients with acquired resistance to cTT. However, response rates are significantly lower than in treatment-naïve patients and might be similar for ipilimumab plus nivolumab and anti-PD-1 monotherapy. The latter raises the question whether trials such as DREAMseq (NCT02224781) and SECOMBIT (NCT02631447) interrogating the optimal sequence of cTT and ICB are appropriately designed to answer this question since they employ combined ICB.

While acquired resistance is the major challenge of cTT, primary refractory disease is a more frequent clinical problem in patients receiving ICB [18]. Several mechanisms have been described that mostly impair interferon sensitivity [42, 43] and/or antigen presentation [44] for anti-PD-1 monotherapy. Recently, association of primary resistance to PD-1 blockade with mutations and losses in JAK1 that can mediate interferon resistance was confirmed [45]. Mechanisms of resistance to combined ipilimumab and nivolumab remain elusive but might be different from those observed in patients receiving pembrolizumab or nivolumab [46, 47]. As stated above for cTT, primary resistance to ICB cannot be overcome specifically, but several trials are evaluating new strategies to reach this goal. Cross-resistance to cTT in patients not responding to ICB might exist. Clinical data show that activity of second-line cTT remains high, but PFS might be shorter than in treatment-naïve patients [40, 48].

\section{Summary/Discussion}

To date, patients with unresectable or metastatic melanoma, harboring an activating mutation in the $B R A F$ gene, have two different treatment options. As described above, the long-term follow-up data for cTT show promising results, with a 5-year landmark OS of $34 \%$ on treatment with D+T in patients harboring a $B R A F \mathrm{~V} 600 \mathrm{E} / \mathrm{K}$ mutation. When looking at the survival outcome for patients with the most favorable baseline characteristics (i.e. normal LDH levels, ECOG PS 0, low tumor burden, no evidence of MBMs) and a CR to $\mathrm{D}+\mathrm{T}$, the 5-year landmark OS rate reached up to $71 \%$. In comparison, patients with a $B R A F$ mutation receiving a combined ICB with ipilimumab and nivolumab show a 5 -year survival rate of $60 \%$ without incorporating additional favorable baseline characteristics. Certainly, these results indicate that long-term survival of BRAF V600-mutated melanoma is possible, both with cTT and ICB. To evaluate if these data indicate a true cure for metastatic melanoma, we first have to define what achieving a 'true cure' means.

Appendicitis is cured surgically and cellulitis is cured with antibiotics. In contrast, diabetes and psoriasis are managed, treated, or controlled. The goal of treating patients with metastatic melanoma or other advanced malignancies is to provide palliation by disease control and an extension of the life expectancy without decreasing the quality of life (QoL) by treatment-related AEs. Therefore, a true cure for melanoma means that there is no evidence of the disease and no relapse will ever occur, ideally without continuous treatment. In this perspective, non-adjuvant cTT seems unable to reach this goal. Given the low and still dropping rate of patients without progression after 5 years of cTT, it seems unlikely that this approach will cure patients. In addition, approximately $50 \%$ of melanoma patients undergoing cTT will relapse/progress if treatment is discontinued, indicating that clinical activity of these drugs is tightly associated with continuous exposure [49]. In contrast, ICB can provide clinical benefit even after discontinuation of treatment [50]. These notions might change when cTT is used adjuvantly: statistical models imply that 12 months of adjuvant cTT with $\mathrm{D}+\mathrm{T}$ provide sustained benefit to patients.

In 2018, the FDA and EMA approved the use of dabrafenib plus trametinib in an adjuvant setting for stage III melanoma. Hauschild et al. presented extended followup data regarding the relapse-free survival (RFS) of the COMBI-AD study (NCT01682083), a randomized, phase III trial, where patients with resected BRAF V600-mutated stage III melanoma were assigned to 12 months of $\mathrm{D}+\mathrm{T}$ versus placebo [51]. Here, at a median follow-up of 44 months, patients receiving the cTT showed a 4-year RFS rate of 54\% (95\% CI 49-59). An estimated statistical cure rate of $54 \%$ (95\% CI 49-59) for cTT, compared with 37\% (95\% CI $32-42$ ) in the placebo group, has also been described. These results increase the hope for a real 'cure' by cTT, but longterm and OS data are still pending.

Since data indicate that curing patients with advanced $B R A F$ V600E/K-mutated melanoma using non-adjuvant cTT is unlikely in the overall patient population, it becomes very important to choose the most suitable first-line therapeutic option. Very recently, a 5-year analysis of the pivotal CheckMate 067 trial, performed by Larkin et al., showed a survival benefit for patients with a $B R A F$ mutation undergoing combined ICB in comparison to $B R A F$ wild-type patients [18]. Interestingly, patients harboring a $B R A F$ V600 mutation 
showed a 5-year OS rate of $60 \%$, whereas $B R A F$ wild-type patients reached a 5 -year OS rate of $46 \%$. While these findings might have been influenced by second-line therapy, the median PFS of 11.2 months (95\% CI 7.0-18.1) for $B R A F$ wild-type patients and 16.8 months (95\% CI 8.3-32.0) for $B R A F$-mutated patients, also indicates a direct impact of mutation status on ICB outcomes. In this trial, the median OS for patients receiving nivolumab monotherapy and harboring a BRAF mutation was 45.5 months (95\% CI 26.4-not reached), compared with 34.4 months (95\% CI 24.1-59.2) for patients without a $B R A F$ mutation. The median PFS rates for both cohorts were 5.6 months (95\% CI 2.8-9.5) and 8.2 months (95\% CI 5.1-19.6), respectively.

Considering an additional subgroup analysis from CheckMate 067 showing a 64\% survival rate in all patients with normal baseline LDH and less than three organ sites with metastases, it is likely that combined ICB might show a 5-year survival rate $>64 \%$ in $B R A F$-mutated patients showing additional favorable baseline characteristics. Although these results should be interpreted with great caution, an indirect comparison by Atkins et al. showed improved survival outcome in $B R A F$-mutated melanoma patients receiving ipilimumab plus nivolumab compared with $\mathrm{V}+\mathrm{C}$ and $\mathrm{D}+\mathrm{T}$ [52]. This is also supported by indirectly comparing the 5-year landmark OS rates of COMBI-v/d and CheckMate 067 (34\% vs. $60 \%$ ). These findings are also in line with the results of a retrospective study [53] in which patients with a $B R A F$ mutation treated with an anti-PD-1 monotherapy as first-line therapy had longer OS compared with patients treated with first-line cTT. Another retrospective analysis included 567 BRAF-mutated patients with advanced disease stage who received first-line therapy of either cTT $(n=297$; $n=262 \mathrm{D}+\mathrm{T}$ and $n=35 \mathrm{~V}+\mathrm{C}$ ), anti-PD-1 monotherapy ( $n=162 ; n=69$ nivolumab and $n=93$ pembrolizumab), or combined ICB ( $n=108$ ipilimumab plus nivolumab) [54]. After a median follow-up of 22.4 months (interquartile range [IQR] 10.3-32.7), the median OS was 13.2 months (IQR 5.2-41.4), 39.5 months (IQR 8.7-not reached), and not reached (IQR 8.7-not reached), respectively. A 36-month follow-up of the CheckMate 067 trial showed similar results, with a median OS for nivolumab monotherapy of 37.6 months (95\% CI 29.1-not reached) and not reached (95\% CI 38.2 months-not reached) for ipilimumab plus nivolumab in the entire study population [55]. In contrast, the presented median OS for first-line cTT was significantly lower than for patients treated within the COMBI-d trial (median OS 25.1 months; 95\% CI 19.2-not reached) [56]). A possible explanation for this difference could be the less favorable baseline characteristics in the real-world setting (e.g. presence of MBMs, elevated LDH levels), as indicated by our own studies $[38,53]$.

Another retrospective study analyzed the treatment outcome for ipilimumab plus nivolumab as first-line therapy or after not responding to cTT or anti-PD-1 ICB in advanced (unresectable stage IIIC/IV) melanoma in a real-world setting [39]. A total of 152 patients were included $(n=60$ first-line; $n=33$ s-line after cTT; $n=57$ s-line after antiPD-1 ICB). After a median follow-up of 6.1 months (95\% CI 5.8-6.7), the median OS for first-line ipilimumab plus nivolumab was 14.2 months (95\% CI 8.0-not reached), compared with 3.6 months (95\% CI 1.6-not reached) for second-line after not responding to cTT and 9.6 months (95\% CI 7.8-not reached) for second-line after not responding to anti-PD-1 monotherapy. Of all patients included in that study, 26\% had MBMs, 55\% had elevated LDH levels, and $39 \%$ had ECOG PS $\geq 1$, explaining a less favorable treatment outcome compared with the results of the CheckMate 067 trial. Coming back to biomarkers associated with favorable outcomes, these and other real-life data [38] show an accumulation of patients with unfavorable baseline characteristics among those receiving first-line cTT. Out of clinical practice, patients presenting with a high tumor burden and/ or symptomatic brain metastases are especially in need of immediate disease control. Therefore, patients presenting with these characteristics and harboring a BRAF mutation seem to be preferentially treated with first-line cTT, since palliation by disease control is the primary goal, even if ICB might be superior in the long run. Nonetheless, no definitive recommendations can be made due to the lack of direct comparison of ICB and TT in clinical trials.

Apart from efficacy, safety and QoL are major features of clinical importance. Regarding QoL, prospective clinical trials have shown that cTT and ICB have no significant impact on the QoL of melanoma patients, as previously reviewed by Malkhasyan et al. [57]; however, relapse and progression are associated with a drop in QoL. Many AEs have been described for cTT and ICB. In patients treated with cTT, AEs of any grade (according to the Common Terminology Criteria for Adverse Events [CTCAE]) occurred in almost all patients, with grade $3-4$ AEs occurring in $54 \% / 62 \%$ of patients for $\mathrm{D}+\mathrm{T}$ (COMBI-d, COMBI-v [4]), $60 \%$ for $\mathrm{V}+\mathrm{C}$ (coBRIM [13]), and 58\% for E + B (COLUMBUS, Part 1 [12]). In CheckMate 067, combined ICB caused grade 3-4 toxicities in 59\% of patients, while anti-PD-1 monotherapy led to grade 3-4 AEs in 23\% of patients [16]. Importantly, long-term exposure to cTT and/or long-term follow-up in patients receiving or having received ICB are not associated with new AEs. In general, the incidence of AEs is dropping with longer follow-up.

Taken together, is there a hope for cure? Yes, certainly. Under favorable circumstances (i.e. low LDH levels at baseline, few metastatic organ sites, no brain metastases, $B R A F$ $\mathrm{V} 600 \mathrm{E} / \mathrm{K}$ mutation), there is a good chance of long disease control with ongoing PFS and high OS in patients receiving first-line cTT or ICB for the treatment of metastatic disease. As stated above, a true cure means that patients are without 
a relapse 'forever'. Two aspects must be considered in this perspective: how long do we have to follow-up patients for relapses and at which point in time do we have to document melanoma-specific survival instead of OS? At present, answering these questions based on evidence is impossible.

With head-to-head data pending, indirect comparison and interpretation of results from prospective studies evaluating first-line ICB in melanoma patients harboring a BRAF V600 mutation indicate that long-term disease control is more likely in patients receiving first-line ICB, particularly ipilimumab plus nivolumab. However, apart from these considerations, concurrent medical conditions, symptoms, shared decision making, and other factors have to be incorporated into the treatment of patients with advanced melanoma.

Acknowledgements Open Access funding provided by Projekt DEAL.

\section{Compliance with Ethical Standards}

Funding No funding was received to assist in the preparation of this review.

Conflict of interest Patrick Schummer has received honoraria from Bristol-Myers Squibb (BMS), an institutional research grant from Novartis, and reports travel support from Novartis, Lilly and BMS outside the submitted work. Bastian Schilling reports grants and personal fees from BMS, MSD Sharp \& Dohme and Pierre Fabre Pharmaceuticals, and personal fees from Incyte, Novartis, Roche, Pfizer and AMGEN outside the submitted work. Anja Gesierich reports advisory roles for BMS, MSD Sharp \& Dohme, Novartis, Roche, Pierre Fabre Pharmaceuticals, Pfizer and Sanofi Genzyme, has received honoraria from MSD Sharp \& Dohme, BMS and Roche, and travel support from BMS, MSD Sharp \& Dohme, Novartis, Roche and Pierre Fabre Pharmaceuticals outside of the submitted work.

Open Access This article is licensed under a Creative Commons Attribution-NonCommercial 4.0 International License, which permits any non-commercial use, sharing, adaptation, distribution and reproduction in any medium or format, as long as you give appropriate credit to the original author(s) and the source, provide a link to the Creative Commons licence, and indicate if changes were made. The images or other third party material in this article are included in the article's Creative Commons licence, unless indicated otherwise in a credit line to the material. If material is not included in the article's Creative Commons licence and your intended use is not permitted by statutory regulation or exceeds the permitted use, you will need to obtain permission directly from the copyright holder.To view a copy of this licence, visit http://creativecommons.org/licenses/by-nc/4.0/.

\section{References}

1. Siegel RL, Miller KD, Jemal A. Cancer statistics, 2019. CA Cancer J Clin. 2019;69(1):7-34. https://doi.org/10.3322/caac.21551.

2. Hodis E, Watson IR, Kryukov GV, Arold ST, Imielinski M, Theurillat JP, et al. A landscape of driver mutations in melanoma. Cell. 2012;150(2):251-63. https://doi.org/10.1016/j. cell.2012.06.024.
3. Davies H, Bignell GR, Cox C, Stephens P, Edkins S, Clegg S, et al. Mutations of the BRAF gene in human cancer. Nature. 2002;417(6892):949-54. https://doi.org/10.1038/nature00766.

4. Robert C, Grob JJ, Stroyakovskiy D, Karaszewska B, Hauschild A, Levchenko E, et al. Five-year outcomes with dabrafenib plus trametinib in metastatic melanoma. N Engl J Med. 2019;381(7):626-36. https://doi.org/10.1056/NEJMoa1904059.

5. Amaral T, Sinnberg T, Meier F, Krepler C, Levesque M, Niessner $\mathrm{H}$, et al. The mitogen-activated protein kinase pathway in melanoma part I-activation and primary resistance mechanisms to BRAF inhibition. Eur J Cancer. 2017;73:85-92. https://doi. org/10.1016/j.ejca.2016.12.010.

6. Amaral T, Sinnberg T, Meier F, Krepler C, Levesque M, Niessner $\mathrm{H}$, et al. MAPK pathway in melanoma part II-secondary and adaptive resistance mechanisms to BRAF inhibition. Eur J Cancer. 2017;73:93-101. https://doi.org/10.1016/j.ejca.2016.12.012.

7. Yu Q, Xie J, Li J, Lu Y, Liao L. Clinical outcomes of BRAF plus MEK inhibition in melanoma: a meta-analysis and systematic review. Cancer Med. 2019;8(12):5414-24. https://doi. org/10.1002/cam4.2248.

8. Shi H, Hugo W, Kong X, Hong A, Koya RC, Moriceau G, et al. Acquired resistance and clonal evolution in melanoma during BRAF inhibitor therapy. Cancer Discov. 2014;4(1):80-93. https ://doi.org/10.1158/2159-8290.CD-13-0642.

9. Long GV, Stroyakovskiy D, Gogas H, Levchenko E, de Braud F, Larkin J, et al. Combined BRAF and MEK inhibition versus BRAF inhibition alone in melanoma. N Engl $\mathrm{J}$ Med. 2014;371(20):1877-88. https://doi.org/10.1056/NEJMoa1406037.

10. Robert C, Karaszewska B, Schachter J, Rutkowski P, Mackiewicz A, Stroiakovski D, et al. Improved overall survival in melanoma with combined dabrafenib and trametinib. N Engl J Med. 2015;372(1):30-9. https://doi.org/10.1056/NEJMoa1412690.

11. Larkin J, Ascierto PA, Dreno B, Atkinson V, Liszkay G, Maio M, et al. Combined vemurafenib and cobimetinib in BRAF-mutated melanoma. N Engl J Med. 2014;371(20):1867-76. https://doi. org/10.1056/NEJMoa1408868.

12. Dummer R, Ascierto PA, Gogas HJ, Arance A, Mandala M, Liszkay $\mathrm{G}$, et al. Encorafenib plus binimetinib versus vemurafenib or encorafenib in patients with BRAF-mutant melanoma (COLUMBUS): a multicentre, open-label, randomised phase 3 trial. Lancet Oncol. 2018;19(5):603-15. https://doi.org/10.1016/S1470 -2045(18)30142-6.

13. Ascierto PA, McArthur GA, Dreno B, Atkinson V, Liszkay G, Di Giacomo AM, et al. Cobimetinib combined with vemurafenib in advanced BRAF(V600)-mutant melanoma (coBRIM): updated efficacy results from a randomised, double-blind, phase 3 trial. Lancet Oncol. 2016;17(9):1248-60. https://doi.org/10.1016/ S1470-2045(16)30122-X.

14. Hauschild A, Larkin J, Ribas A, Dreno B, Flaherty KT, Ascierto PA, et al. Modeled prognostic subgroups for survival and treatment outcomes in BRAF V600-mutated metastatic melanoma: pooled analysis of 4 randomized clinical trials. JAMA Oncol. 2018;4(10):1382-8. https://doi.org/10.1001/jamao ncol.2018.2668.

15. Dummer R, Ascierto PA, Gogas HJ, Arance A, Mandala M, Liszkay $\mathrm{G}$, et al. Overall survival in patients with BRAF-mutant melanoma receiving encorafenib plus binimetinib versus vemurafenib or encorafenib (COLUMBUS): a multicentre, open-label, randomised, phase 3 trial. Lancet Oncol. 2018;19(10):1315-27. https://doi.org/10.1016/S1470-2045(18)30497-2.

16. Hamid O, Robert C, Daud A, Hodi FS, Hwu WJ, Kefford R, et al. Five-year survival outcomes for patients with advanced melanoma treated with pembrolizumab in KEYNOTE-001. Ann Oncol. 2019;30(4):582-8. https://doi.org/10.1093/annonc/mdz011.

17. Robert C, Ribas A, Schachter J, Arance A, Grob JJ, Mortier L, et al. Pembrolizumab versus ipilimumab in advanced melanoma 
(KEYNOTE-006): post-hoc 5-year results from an open-label, multicentre, randomised, controlled, phase 3 study. Lancet Oncol. 2019;20(9):1239-51. https://doi.org/10.1016/S1470 -2045(19)30388-2.

18. Larkin J, Chiarion-Sileni V, Gonzalez R, Grob JJ, Rutkowski P, Lao CD, et al. Five-year survival with combined nivolumab and ipilimumab in advanced melanoma. N Engl J Med. 2019. https:// doi.org/10.1056/NEJMoa1910836.

19. Larkin J, Minor D, D’Angelo S, Neyns B, Smylie M, Miller WH $\mathrm{Jr}$, et al. Overall survival in patients with advanced melanoma who received nivolumab versus investigator's choice chemotherapy in CheckMate 037: a randomized, controlled, open-label phase III trial. J Clin Oncol. 2018;36(4):383-90. https://doi.org/10.1200/ JCO.2016.71.8023.

20. Dickson PV, Gershenwald JE. Staging and prognosis of cutaneous melanoma. Surg Oncol Clin N Am. 2011;20(1):1-17. https://doi. org/10.1016/j.soc.2010.09.007.

21. Gershenwald JE, Scolyer RA, Hess KR, Sondak VK, Long GV, Ross MI, et al. Melanoma staging: evidence-based changes in the American Joint Committee on Cancer eighth edition cancer staging manual. CA Cancer J Clin. 2017;67(6):472-92. https://doi. org/10.3322/caac.21409.

22. Sperduto PW, Kased N, Roberge D, Xu Z, Shanley R, Luo X, et al. Summary report on the graded prognostic assessment: an accurate and facile diagnosis-specific tool to estimate survival for patients with brain metastases. J Clin Oncol. 2012;30(4):419-25. https:// doi.org/10.1200/JCO.2011.38.0527.

23. Davies MA, Saiag P, Robert C, Grob JJ, Flaherty KT, Arance A, et al. Dabrafenib plus trametinib in patients with BRAF(V600)-mutant melanoma brain metastases (COMBI$\mathrm{MB})$ : a multicentre, multicohort, open-label, phase 2 trial. Lancet Oncol. 2017;18(7):863-73. https://doi.org/10.1016/S1470 -2045(17)30429-1.

24. Long GV, Atkinson V, Lo S, Sandhu S, Guminski AD, Brown MP, et al. Combination nivolumab and ipilimumab or nivolumab alone in melanoma brain metastases: a multicentre randomised phase 2 study. Lancet Oncol. 2018;19(5):672-81. https://doi.org/10.1016/ S1470-2045(18)30139-6.

25. Tawbi HA, Forsyth PA, Algazi A, Hamid O, Hodi FS, Moschos $\mathrm{SJ}$, et al. Combined nivolumab and ipilimumab in melanoma metastatic to the brain. N Engl J Med. 2018;379(8):722-30. https ://doi.org/10.1056/NEJMoa1805453.

26. Lewis KD, Larkin J, Ribas A, Flaherty KT, McArthur GA, Ascierto PA, et al. Impact of depth of response on survival in patients treated with cobimetinib +/- vemurafenib: pooled analysis of BRIM-2, BRIM-3, BRIM-7 and coBRIM. Br J Cancer. 2019;121(7):522-8. https://doi.org/10.1038/s41416-019-0546-y.

27. Menzer C, Menzies AM, Carlino MS, Reijers I, Groen EJ, Eigentler T, et al. Targeted therapy in advanced melanoma with rare BRAF mutations. J Clin Oncol. 2019;37(33):3142-51. https ://doi.org/10.1200/JCO.19.00489.

28. Wang J, Chen J, Miller DD, Li W. Synergistic combination of novel tubulin inhibitor ABI-274 and vemurafenib overcome vemurafenib acquired resistance in BRAFV600E melanoma. Mol Cancer Ther. 2014;13(1):16-26. https://doi.org/10.1158/1535-7163. MCT-13-0212.

29. Shaffer SM, Dunagin MC, Torborg SR, Torre EA, Emert B, Krepler C, et al. Rare cell variability and drug-induced reprogramming as a mode of cancer drug resistance. Nature. 2017;546(7658):431-5. https://doi.org/10.1038/nature22794.

30. Nazarian R, Shi H, Wang Q, Kong X, Koya RC, Lee H, et al. Melanomas acquire resistance to B-RAF(V600E) inhibition by RTK or N-RAS upregulation. Nature. 2010;468(7326):973-7. https://doi.org/10.1038/nature09626.

31. Yadav V, Zhang X, Liu J, Estrem S, Li S, Gong XQ, et al. Reactivation of mitogen-activated protein kinase (MAPK) pathway by FGF receptor 3 (FGFR3)/Ras mediates resistance to vemurafenib in human B-RAF V600E mutant melanoma. J Biol Chem. 2012;287(33):28087-988. https://doi.org/10.1074/jbc. M112.377218.

32. Poulikakos PI, Persaud Y, Janakiraman M, Kong X, Ng C, Moriceau $\mathrm{G}$, et al. RAF inhibitor resistance is mediated by dimerization of aberrantly spliced BRAF(V600E). Nature. 2011;480(7377):387-90. https://doi.org/10.1038/nature10662.

33. Choi J, Landrette SF, Wang T, Evans P, Bacchiocchi A, Bjornson R, et al. Identification of PLX4032-resistance mechanisms and implications for novel RAF inhibitors. Pigment Cell Melanoma Res. 2014;27(2):253-62. https://doi.org/10.1111/ pcmr.12197.

34. Atefi M, von Euw E, Attar N, Ng C, Chu C, Guo D, et al. Reversing melanoma cross-resistance to BRAF and MEK inhibitors by co-targeting the AKT/mTOR pathway. PLoS One. 2011;6(12):e28973. https://doi.org/10.1371/journal.pone.00289 73.

35. Paraiso KH, Xiang Y, Rebecca VW, Abel EV, Chen YA, Munko $\mathrm{AC}$, et al. PTEN loss confers BRAF inhibitor resistance to melanoma cells through the suppression of BIM expression. Cancer Res. 2011;71(7):2750-60. https://doi.org/10.1158/0008-5472. CAN-10-2954.

36. Hugo W, Shi H, Sun L, Piva M, Song C, Kong X, et al. Nongenomic and immune evolution of melanoma acquiring MAPKi resistance. Cell. 2015;162(6):1271-85. https://doi.org/10.1016/j. cell.2015.07.061.

37. Hugo W, Zaretsky JM, Sun L, Song C, Moreno BH, Hu-Lieskovan $\mathrm{S}$, et al. Genomic and transcriptomic features of response to antiPD-1 therapy in metastatic melanoma. Cell. 2016;165(1):35-44. https://doi.org/10.1016/j.cell.2016.02.065.

38. Kreft S, Gesierich A, Eigentler T, Franklin C, Valpione S, Ugurel $\mathrm{S}$, et al. Efficacy of PD-1-based immunotherapy after radiologic progression on targeted therapy in stage IV melanoma. Eur J Cancer. 2019;116:207-15. https://doi.org/10.1016/j.ejca.2019.05.015.

39. Mason R, Dearden HC, Nguyen B, Oon JS, Smith JL, Randhawa $\mathrm{M}$, et al. Combined ipilimumab and nivolumab first-line and after BRAF targeted therapy in advanced melanoma. Pigment Cell Melanoma Res. 2020;33(2):358-65. https://doi.org/10.1111/ pcmr.12831

40. Ackerman A, Klein O, McDermott DF, Wang W, Ibrahim N, Lawrence DP, et al. Outcomes of patients with metastatic melanoma treated with immunotherapy prior to or after BRAF inhibitors. Cancer. 2014;120(11):1695-701. https://doi.org/10.1002/ cncr.28620.

41. Czarnecka AM, Teterycz P, Mariuk-Jarema A, Lugowska I, Rogala P, Dudzisz-Sledz M, et al. Treatment sequencing and clinical outcomes in BRAF-positive and BRAF-negative unresectable and metastatic melanoma patients treated with new systemic therapies in routine practice. Target Oncol. 2019;14(6):729-42. https://doi.org/10.1007/s11523-019-00688-8.

42. Shin DS, Zaretsky JM, Escuin-Ordinas H, Garcia-Diaz A, HuLieskovan S, Kalbasi A, et al. Primary resistance to PD-1 blockade mediated by JAK1/2 mutations. Cancer Discov. 2017;7(2):188201. https://doi.org/10.1158/2159-8290.CD-16-1223.

43. Sucker A, Zhao F, Pieper N, Heeke C, Maltaner R, Stadtler N, et al. Acquired IFNgamma resistance impairs anti-tumor immunity and gives rise to T-cell-resistant melanoma lesions. Nat Commun. 2017;8:15440. https://doi.org/10.1038/ncomms15440.

44. Sade-Feldman M, Jiao YJ, Chen JH, Rooney MS, Barzily-Rokni M, Eliane JP, et al. Resistance to checkpoint blockade therapy through inactivation of antigen presentation. Nat Commun. 2017;8(1):1136. https://doi.org/10.1038/s41467-017-01062-w.

45. Liu D, Schilling B, Liu D, Sucker A, Livingstone E, Jerby-Amon $\mathrm{L}$, et al. Integrative molecular and clinical modeling of clinical outcomes to PD1 blockade in patients with metastatic melanoma. 
Nat Med. 2019;25(12):1916-27. https://doi.org/10.1038/s4159 1-019-0654-5.

46. Zimmer L, Apuri S, Eroglu Z, Kottschade LA, Forschner A, Gutzmer R, et al. Ipilimumab alone or in combination with nivolumab after progression on anti-PD-1 therapy in advanced melanoma. Eur J Cancer. 2017;75:47-55. https://doi. org/10.1016/j.ejca.2017.01.009.

47. Shukla SA, Bachireddy P, Schilling B, Galonska C, Zhan Q, Bango $\mathrm{C}$, et al. Cancer-germline antigen expression discriminates Clinical outcome to CTLA-4 blockade. Cell. 2018;173(3):624-33. https://doi.org/10.1016/j.cell.2018.03.026 (e8).

48. Saab KR, Mooradian MJ, Wang DY, Chon J, Xia CY, Bialczak A, et al. Tolerance and efficacy of BRAF plus MEK inhibition in patients with melanoma who previously have received programmed cell death protein 1-based therapy. Cancer. 2019;125(6):884-91. https://doi.org/10.1002/cncr.31889.

49. Carlino MS, Vanella V, Girgis C, Giannarelli D, Guminski A, Festino L, et al. Cessation of targeted therapy after a complete response in BRAF-mutant advanced melanoma: a case series. Br J Cancer. 2016;115(11):1280-4. https://doi.org/10.1038/ bjc. 2016.321.

50. Robert C, Ribas A, Hamid O, Daud A, Wolchok JD, Joshua $\mathrm{AM}$, et al. Durable complete response after discontinuation of pembrolizumab in patients with metastatic melanoma. $\mathrm{J}$ Clin Oncol. 2018;36(17):1668-744. https://doi.org/10.1200/ JCO.2017.75.6270.

51. Hauschild A, Dummer R, Schadendorf D, Santinami M, Atkinson V, Mandala M, et al. Longer follow-up confirms relapsefree survival benefit with adjuvant dabrafenib plus trametinib in patients with resected BRAF V600-mutant stage III melanoma. J Clin Oncol. 2018;36(35):JCO1801219. https://doi.org/10.1200/ JCO.18.01219.

52. Atkins MB, Tarhini A, Rael M, Gupte-Singh K, O’Brien E, Ritchings C, et al. Comparative efficacy of combination immunotherapy and targeted therapy in the treatment of BRAF-mutant advanced melanoma: a matching-adjusted indirect comparison. Immunotherapy. 2019;11(7):617-29. https://doi.org/10.2217/ imt-2018-0208.

53. Schilling B, Martens A, Geukes Foppen MH, Gebhardt C, Hassel JC, Rozeman EA, et al. First-line therapy-stratified survival in BRAF-mutant melanoma: a retrospective multicenter analysis. Cancer Immunol Immunother. 2019;68(5):765-72. https://doi. org/10.1007/s00262-019-02311-1.

54. Moser JC, Chen D, Hu-Lieskovan S, Grossmann KF, Patel S, Colonna SV, et al. Real-world survival of patients with advanced BRAF V600 mutated melanoma treated with front-line BRAF/ MEK inhibitors, anti-PD-1 antibodies, or nivolumab/ipilimumab. Cancer Med. 2019;8(18):7637-43. https://doi.org/10.1002/ cam4.2625.

55. Wolchok JD, Chiarion-Sileni V, Gonzalez R, Rutkowski P, Grob JJ, Cowey CL, et al. Overall survival with combined nivolumab and ipilimumab in advanced melanoma. N Engl J Med. 2017;377(14):1345-56. https://doi.org/10.1056/NEJMoa1709684.

56. Long GV, Stroyakovskiy D, Gogas H, Levchenko E, de Braud F, Larkin J, et al. Dabrafenib and trametinib versus dabrafenib and placebo for Val600 BRAF-mutant melanoma: a multicentre, double-blind, phase 3 randomised controlled trial. Lancet. 2015;386(9992):444-51. https://doi.org/10.1016/S0140 -6736(15)60898-4.

57. Malkhasyan KA, Zakharia Y, Milhem M. Quality-of-life outcomes in patients with advanced melanoma: a review of the literature. Pigment Cell Melanoma Res. 2017;30(6):511-20. https://doi. org/10.1111/pcmr.12647.

58. Larkin J, Chiarion-Sileni V, Gonzalez R, Grob JJ, Cowey CL, Lao $\mathrm{CD}$, et al. Combined nivolumab and ipilimumab or monotherapy in untreated melanoma. N Engl J Med. 2015;373(1):23-34. https ://doi.org/10.1056/NEJMoa1504030.

59. Ascierto PA, Long GV, Robert C, Brady B, Dutriaux C, Di Giacomo AM, et al. Survival outcomes in patients with previously untreated BRAF wild-type advanced melanoma treated with nivolumab therapy: three-year follow-up of a randomized phase 3 trial. JAMA Oncol. 2019;5(2):187-94. https://doi.org/10.1001/ jamaoncol.2018.4514.

60. Carlino MS, Long GV, Schadendorf D, Robert C, Ribas A, Richtig E, et al. Outcomes by line of therapy and programmed death ligand 1 expression in patients with advanced melanoma treated with pembrolizumab or ipilimumab in KEYNOTE-006: a randomised clinical trial. Eur J Cancer. 2018;101:236-43. https:// doi.org/10.1016/j.ejca.2018.06.034. 